\title{
Dispersion and stabilization of cochleate nanoparticles
}

Tamás Bozó $^{a}$, András Wacha ${ }^{b}$, Judith Mihály ${ }^{b}$, Attila Bóta $^{b}$, Miklós, S. Z. Kellermayer ${ }^{a, c *}$

${ }^{\mathrm{a}}$ Semmelweis University, Department of Biophysics and Radiation Biology, 1094 Budapest, Tüzoltó u. 37-47., Hungary

${ }^{\mathrm{b}}$ Biological Nanochemistry Research Group, Institute of Materials and Environmental Chemistry, Research Centre for Natural Sciences, Hungarian Academy of Sciences, 1117 Budapest, Hungary, Magyar tudósok körútja 2.

cMTA-SE Molecular Biophysics Research Group, Semmelweis University, 1094 Budapest, Hungary, Tüzoltó u. 37-47.

*corresponding author: kellermayer.miklos@med.semmelweis-univ.hu

further e-mail addresses:

T.B.: bozo.tamas@med.semmelweis-univ.hu

A.W.:wacha.andras@ttk.mta.hu

J.M.: mihaly.judith@ttk.mta.hu

A.B.: bota.attila@ttk.mta.hu 


\begin{abstract}
Cochleates, calcium-stabilized membrane rolls of nanoscale diameter, promise a unique and efficient way of delivering lipid-soluble drugs, proteins or nucleic acids into biological systems because they protect the encapsulated material against enzymatic or chemical degradation. Self-aggregation, which typically arises during production and storage is a major obstacle that has so far precluded the development of an efficient cochleate-based drugdelivery system. Here we show that citric acid, added transiently in a narrow concentration range, effectively disperses cochleate aggregates, stabilizes the disperse state for long-term storage and preserves the canonical ultrastructure and topological characteristics of cochleate nanoparticles.
\end{abstract}

Keywords: cochleate, citrate, aggregation, stabilization, calcium, AFM, SAXS, FTIR 


\section{Introduction}

Cochleates are biocompatible, prospective drug-delivery vehicles. They are formed of spirally rolled bilayer sheets of negatively charged phospholipids stabilized by $\mathrm{Ca}^{2+}$ ions [1] so that the lipid-to-calcium binding ratio is thought to be $2: 1$ [1-3]. Several studies have shown their applicability as vaccine adjuvants [4-8] and carriers of antibiotics [9-12], systemic antimycotic agents [13-16] or factor VII for replacement in hemophilia A [17, 18]. However, their widespread use is severely hindered by a major technological obstacle: $\mathrm{Ca}^{2+}$ ions, which are essential in the formation and maintenance of cochleate structure, consistently provoke aggregation of the particles. Aggregation is a serious drawback as it prevents the efficient separation from byproducts, the assessment and monitoring of cochleate formation, the modification and functionalization of cochleate surface and the manufacturing of a disperse, long-shelf-life pharmaceutical delivery system. A recent patent claimed to remedy this problem by adding half and half, milk, casein, albumin or methylcellulose [19]. However, the use of protein excipients may lead to aggravated problems of reduced stability and safety, and altered pharmacokinetics. Furthermore, methylcellulose caused only a partial disruption of aggregates. A long list of other polymeric materials (e.g., celluloses, gums, starches, etc.) has also been suggested as potential aggregation inhibitors, but feasibility is uncertain [19]. A very recent comparison between a novel, microfluidics-based strategy and conventional cochleate production methods has underlined that aggregation prevails as a major, unsolved problem [20]. In the present work we developed an efficient method of preparing stable, disperse cochleates. Dioleoyl phosphatidylserine liposomes, which are the precursors of cochleates, exhibit outstanding colloidal stability. However, upon the addition of $\mathrm{Ca}^{2+}$ they quickly form aggregates. Aggregation is caused by the surface-charge-modifying effect of $\mathrm{Ca}^{2+}[21]$. Therefore, by removing calcium the aggregates are expected to disassemble into their component nanoparticles. Removal of calcium, however, may lead to 
unfavorable consequences, because it is the $\mathrm{Ca}^{2+}$ ions themselves that stabilize the inner structure of cochleates. Indeed, it has been known since the discovery of cochleates that upon the addition of EDTA, a strong chelator of calcium, cochleate particles loose their rolled-up structure and fuse into giant vesicles [1]. We hypothesized that a molecule with an affinity to $\mathrm{Ca}^{2+}$ lower than that of EDTA may be effective to disperse aggregates by removing $\mathrm{Ca}^{2+}$ ions from the outer surface of cochleates but leaving their inner structure intact. To test this hypothesis, we systematically examined sodium salts of mono-, bi- and tricarboxylic acids: acetate, tartrate and citrate. All of these acids are in their fully deprotonated form at $\mathrm{pH} 7.4$, and their association constant to $\mathrm{Ca}^{2+}$ increases with the number of their carboxyl groups (Table 1.).

We show that the addition of sodium citrate in a narrow concentration regime can fully disperse aggregated cochleates, and after citrate removal a colloidally stable system is obtained in which the topology and ultrastructure of the nanoparticles are well preserved. 


\section{Materials and methods}

\section{Materials}

Dioleoyl phosphatidylserine (1,2-Dioleoyl-sn-Glycero-3-[Phospho-L-Serine], DOPS) was purchased from Avanti Polar Lipids, Inc. (Alabaster, Alabama, USA). All other chemicals were from Sigma Aldrich Kft. (Budapest, Hungary).

\section{Preparation of lipid vesicles and cochleates}

Thin DOPS lipid film was formed on the wall of a clean glass tube by evaporating the organic solvent from $500 \mu 120 \mathrm{mg} / \mathrm{ml}$ DOPS/chloroform stock solution under a stream of $\mathrm{N}_{2}$. Organic solvent traces were removed by placing the tube in vacuum $(<20 \mathrm{Hgmm})$ for $30 \mathrm{~min}$. The lipid film was then hydrated with $100 \mu$ aliquots of $100 \mathrm{mM}$ TRIS (pH 7.4) to $1 \mathrm{ml}$ final volume while vigorously vortexing. This multilamellar vesicle (MLV) suspension was extruded 41 times through a polycarbonate membrane filter with $100 \mathrm{~nm}$ pore diameter in an AvantiPolar Mini Extruder (Avanti Polar Lipids, Inc., Alabaster, AL, USA). The resultant small unilamellar vesicle (SUV) suspension of $12.4 \mathrm{mM}$ DOPS concentration was diluted 1 to 1 with $100 \mathrm{mM}$ TRIS (pH 7.4), and $400 \mu 1$ of this solution was dialyzed for 24 hours against $100 \mathrm{ml} 6.2 \mathrm{mM} \mathrm{CaCl}_{2}, 100 \mathrm{mM}$ TRIS (pH 7.4). The obtained cochleate suspension had a nominal $6.2 \mathrm{mM}$ concentration for both DOPS and $\mathrm{Ca}^{2+}$. The sample was stored at room temperature until use. The preparatory steps were done at room temperature.

\section{Dispersion of cochleates}

Aqueous solutions of the tested potential dispersing agents (sodium acetate trihydrate, sodium tartrate dihydrate, trisodium citrate dihydrate, disodium edetate) were prepared in a buffer containing $100 \mathrm{mM}$ TRIS ( $\mathrm{pH} 7.4$ ). We added $10 \mu \mathrm{l}$ of the solution to $10 \mu \mathrm{l}$ cochleate sample and mixed intensively by drawing through a narrow pipette tip 50 times. Samples were 
examined within 10 minutes after being mixed with dispersing agents. The actual concentrations of dispersing agents are given in figure captions. Control experiments were carried out with buffer only.

To obtain a stabilized colloidal system, $20 \mu$ of cochleate sample freshly dispersed with citrate was diluted with $80 \mu \mathrm{l}$ of $100 \mathrm{mM}$ TRIS (pH 7.4), then clarified by centrifugation (Heraeus Biofuge Pico, Life Technologies Magyarország Kft., Budapest, Hungary, 12800 x $\mathrm{g}$, at $25 \pm 1{ }^{\circ} \mathrm{C}, 5$ minutes). The supernatant was gently removed and the pellet was resuspended in $90 \mu \mathrm{l}$ of $100 \mathrm{mM}$ TRIS (pH 7.4). The procedure was repeated 3 times. To test the effect of $\mathrm{Ca}^{2+}$ on resuspended cochleates, the pellets were resuspended with $100 \mathrm{mM}$ TRIS (pH 7.4) containing $6.2 \mathrm{mM} \mathrm{CaCl}_{2}$.

\section{Phase contrast microscopy}

Micrographs were recorded with a Nikon Eclipse Ti-U inverted microscope (Auro-Science Kft., Budapest, Hungary) equipped with a uEye UI 1220 LE digital camera (IDS Imaging Development Systems GmbH, Obersulm, Germany) using either a 20x or a 40x Nikon S Planfluor phase contrast objective.

\section{Atomic force microscopy}

For AFM imaging $10 \mu 1$ of the control or dispersed cochleate sample was diluted with $50 \mu 1$ $100 \mathrm{mM}$ TRIS (pH 7.4) then applied to the substrate and, after 1 minute incubation, washed gently with MilliQ water and dried in a stream of $\mathrm{N}_{2}$ gas. For control cochleates freshly cleaved mica sheets were used as substrate. In the case of dispersed cochleates we used mica sheets pre-coated with $100 \mu 10.01 \%$ poly-L-lysine for $20 \mathrm{~min}$. Cochleates were imaged with a Cypher instrument (Asylum Research, Santa Barbara, CA) with 0.5 to $2.5 \mathrm{~Hz}$ line-scanning rate in air at $29 \pm 1^{\circ} \mathrm{C}$. A silicon cantilever (OMCL AC-160TS, Olympus, Japan) was used in 
non-contact mode, oscillated at its resonance frequency (300-320 kHz, typically). Images with $512 \times 512$ pixel dimensions were collected and analyzed with the built-in algorithms of the AFM driver software.

\section{Small-angle X-ray scattering}

Small-angle X-ray scattering (SAXS) measurements on the cochleate samples were carrried out with a CREDO instrument integrated with a GeniX ${ }^{3 D} \mathrm{Cu}$ ULD microfocus system (Xenocs SA, Sassenage, France) for producing X-rays and a Pilatus-300k CMOS hybrid pixel position sensitive detector (Dectris Ltd, Baden, Switzerland) [22, 23]. The recorded 2D scattering patterns were subjected to the standard on-line data reduction procedure of the instrument control software. Corrections were made for instrumental background, detector flatness, and sample self-absorption. The abscissa was calibrated with mesoporous silica into scattering variable units $q=(4 \cdot \pi \cdot \sin \theta) \cdot \lambda^{-1}$, where $2 \theta$ is the scattering angle and $\lambda=0.154 \mathrm{~nm}$ is the $\mathrm{X}$-ray wavelength of $\mathrm{Cu} \mathrm{K \alpha}$ radiation. Intensity was transformed from count rates to differential scattering cross-section $\left(\mathrm{cm}^{-1} \cdot \mathrm{sr}^{-1}\right)$ units using a pre-calibrated glassy carbon specimen.

The MLV sample was used without further processing. Cochleate samples were clarified by centrifugation (see above). All the samples were filled into borosilicate capillaries of cca. 1 $\mathrm{mm}$ outer diameter and $0.01 \mathrm{~mm}$ wall thickness and centrifuged gently (Eppendorf Minispin microcentrifuge, Eppendorf AG, Hamburg, Germany, $67 \mathrm{x}$ g; at $25 \pm 1{ }^{\circ} \mathrm{C}, 5 \mathrm{~min}$ ) to prevent sedimentation during experiment. The total exposure time was at least 2 hours in each case, carried out in several 5-minute exposures in order to monitor sample stability.

\section{Infrared spectroscopy}


ATR-FTIR spectra were collected with a Varian 2000 FTIR Scimitar Series (Varian Inc., Paolo Alto, CA) spectrometer equipped with a 'GoldenGate' (Specac Ltd., London, UK) single reflection diamond ATR accessory. The measurements were performed at room temperature: $5 \mu \mathrm{l}$ sample was mounted on the top of the diamond ATR crystal. DOPS SUV sample was applied directly, while cochleate samples were clarified by centrifugation (see at dispersion of cochleates) and once more centrifuged at $12800 \mathrm{x} \mathrm{g}$ for 5 minutes. The supernatant was removed and the resuspended pellet was used. IR spectra were recorded both on hydrated lipid systems and as dry films obtained on diamond ATR surface after solvent evaporation. We collected 128 scans at a resolution of $2 \mathrm{~cm}^{-1}$. ATR correction was executed after each data collection. All spectral manipulations were performed using the GRAMS/32 software package (Galactic Industries Incorporation, USA). 


\section{Results and Discussion}

\section{Standard cochleate preparation yields aggregates}

Upon the addition of calcium to DOPS vesicles a milky suspension formed that contained micron-size aggregates of irregular shape (Figure 1. A.). Elongated objects identified as cochleates protruded from the aggregates the bulk of which consisted of aggregated vesicles (Figure 1. B and Supplement figure 1.). Cochleates and vesicles were strongly bound to each other and could not be dispersed by either dilution or intense shaking (Supplement figure 2.).

\section{Citrate disperses aggregates}


To test the hypothesis that a molecule with appropriate affinity to $\mathrm{Ca}^{2+}$ may be effective to disperse aggregates, we added different carboxylic acids to cochleate samples. These acids are fully deprotonated at $\mathrm{pH} 7.4$, and their affinity to $\mathrm{Ca}^{2+}$ increases with the number of their carboxyl groups (Table 1.). Acetate did not cause any disassembly of the aggregates even at a concentration of $500 \mathrm{mM}$ (Supplement figure 3. A). Treatment with $500 \mathrm{mM}$ tartrate led only to a partial disassembly (Supplement figure 3. B). By contrast, citrate effectively dispersed the cochleates (Figure 2. A). Furthermore, the dispersing effect of citrate was concentrationdependent (Supplement figure 4.). At the given experimental lipid and $\mathrm{Ca}^{2+}$ concentrations the optimal citrate concentration range was $200-212.5 \mathrm{mM}$. Below this concentration regime aggregates disassembled only partially, while above this regime the cochleates transformed into giant vesicles (Supplement figure 4.). Thus, there is a relatively narrow concentration range in which citric acid functions as an efficient dispersing agent of cochleate-vesicle aggregates.

\section{Assurance of long-term cochleate stability}

Figure 2. B. shows a cochleate sample 24 hours after it was dispersed by citrate. Individual cochleates, which were clearly distinguishable after citrate addition (Figure 2. A), were no longer observed. Rather, they transformed into clusters of giant vesicles. Thus, citrate, even when applied in the optimal dispersing concentration, disintegrates cochleates with time by extracting the calcium ions from in between the membrane layers. To avoid this effect, we removed excess citrate by centrifuging the sample immediately following the complete dispersion of cochleates then resuspended the pellet in $100 \mathrm{mM}$ TRIS (pH 7.4). The cochleates in this sample preserved their structure, and, although sedimented with time, by stirring they could be well dispersed even after one month of storage at $25 \pm 1^{\circ} \mathrm{C}$ (Figure 2 . C). If $\mathrm{Ca}^{2+}$ concentration was restored in the disperse suspension, cochleate aggregation took 
place again (Supplement figure 5.). Thus, by removing $\mathrm{Ca}^{2+}$, citrate treatment altered the surface properties of cochleates and thereby prevented their aggregation.

\section{Citrate treatment preserves the high-resolution structure of cochleates}

To test whether the cylindrical particles found in the citrate-treated samples (Figures 2.A and

C) preserved the canonical cochleate structure, we carried out high-resolution AFM

measurements. We were unable to discern any appreciable structural differences between the control (Figure 1. B.) and citrate-treated cochleate particles (Figure 3. and Supplement figure 6.). Both control and citrate-stabilized cochleates displayed features consistent with their presumed cochleate model $[1,29,30]$. Accordingly, both samples contained elongated particles with gradually varying diameter across their longitudinal axis. The edges of lipid layers were clearly seen on their surface indicating a roll-like arrangement. There was only one striking difference between the samples: whereas in control samples the cochleates were often part of an aggregate composed of roll-like and vesicular structures (Figure 1.B.), in the citrate-treated sample the particles were systematically dispersed (Figure 3. and Supplement figure 6.). Thus, citrate can effectively disperse cochleates without damaging their unique structure.

Although the citrate-treated and control cochleates are topologically essentially identical, ultrastructural differences might be present. To explore the effect of citrate on the internal structure of cochleates we used small-angle x-ray scattering (SAXS) which can reveal the changes in correlation and ordering of vicinal bilayers. Scattering was measured in an interval corresponding to the range of bilayer thickness and periodicity $\left(0.3-6 \mathrm{~nm}^{-1}\right)$. The investigated samples were a DOPS multilamellar vesicle suspension, a control sample with aggregated cochleate particles, another two with $100 \mathrm{mM}$ and $212.5 \mathrm{mM}$ citrate, and finally one containing $50 \mathrm{mM}$ EDTA instead of citrate (Figure 4). Notably, at a citrate concentration 
of $100 \mathrm{mM}$ cochleates were partially dispersed, while at $212.5 \mathrm{mM}$ dispersion was complete (Supplement figure 4.) and the addition of $50 \mathrm{mM}$ EDTA led to the transformation of cochleates into giant vesicles (Supplement figure 7.). The hydrated DOPS system, which is expected to form multilamellar vesicles, shows a very broad signal between $0.5 \mathrm{~nm}^{-1}<\mathrm{q}<3$ $\mathrm{nm}^{-1}$ (Figure 4. A) typical for entirely uncorrelated multilamellar systems [31]. The absence of long-range ordering is caused by the electrostatic repulsion of the neighbouring bilayers due to the net negative charge of the phosphatidylserine headgroups. The addition of $\mathrm{Ca}^{2+}$ caused a drastic change in the layer organization as seen from the sharp, equidistant Braggreflections at 1.2, 2.4 and $3.77 \mathrm{~nm}^{-1}$ (Figure 4. B). The highly ordered and periodic lamellar state corresponds to the cochleate and has a repeat distance of $5.15 \mathrm{~nm}$, similarly to the findings of Nagarsekar et al. [20]. This structure is retained even following the addition of citrate (Figure 4. C and D). The peak positions align well with the control cochleate sample and correspond to a $5.18 \mathrm{~nm}$ repeat distance. The observed loss in intensity was likely caused by the different sedimentation rate of samples which resulted in different amounts of lipid material in the lower region of the capillaries where the X-ray beam was focused. At high citrate concentration the SAXS signal monotonically increases towards small angle intervals, which is attributed to the diffuse scattering by the smaller particles emerging during the disaggregation process (Figure 4. D). This feature was absent in control cochleate (Figure 4. B) and cochleate $+100 \mathrm{mM}$ citrate (Figure 4. C) samples, because the scattering by the large aggregates made little contribution in this region. The addition of EDTA induced significant changes in bilayer structure (Figure 4. E). The overall scattering curve resembles that of uncorrelated bilayers, and a single Bragg peak at $0.92 \mathrm{~nm}^{-1}$ appeared. This reflection corresponds most likely to the small amount of regular multilamellar bilayer stacks present in the system. The periodicity $(6.8 \mathrm{~nm})$ is significantly greater than that in cochleates $(5.15 \mathrm{~nm})$ indicating that layer packing is less tight than in cochleates. In summary, applying citrate at a 
concentration optimal to disperse aggregated cochleates does not modify the inner structure of the membrane rolls.

\section{Citrate removes $\mathrm{Ca}^{2+}$ from cochleate-surface head groups}

To reveal the molecular mechanisms of the dispersing effect of citrate, we carried out infrared spectroscopy measurements with a focus on the phosphate $\left(1250-100 \mathrm{~cm}^{-1}\right.$, Figure 5 . A), methylene (3000-2800 $\mathrm{cm}^{-1}$, Figure 5. B) and ester carbonyl $\left(1760-1690 \mathrm{~cm}^{-1}\right.$, Figure 5. C) stretching vibrations which are sensitive to phosphate head group hydration, acyl-chain order and glycerol-group hydration, respectively.

The asymmetric and symmetric phosphate $\mathrm{PO}_{2}^{-}$stretching bands at 1219 and $1065 \mathrm{~cm}^{-1}$, respectively, were rather featureless for the DOPS SUV system (Figure 5. A, spectrum a). In control cochleates the $\mathrm{PO}_{2}{ }^{-}$asymmetric stretching band shifted from 1219 to $1236 \mathrm{~cm}^{-1}$ and the symmetric $\mathrm{PO}_{2}{ }^{-}$stretching band split to four components $\left(1111,1102,1075\right.$ and $1063 \mathrm{~cm}^{-}$

${ }^{1}$, Figure 5. A, spectrum b). These changes point at the dehydration of the phosphate group and the formation of a bidentate complex between the $\mathrm{Ca}^{2+}$ ion and the phosphate ester [32]. Addition of $100 \mathrm{mM}$ citrate did not cause any spectral changes in the phosphate head group region (Figure 5. A, spectrum c). At $212.5 \mathrm{mM}$ citrate concentration the relative intensities of phosphate stretching bands, characteristic to $\mathrm{Ca}^{2+}$-DOPS interaction, decreased (Figure 5. A, spectrum d), implying that a fraction of $\mathrm{Ca}^{2+}$-phosphate complexes had broken up. Addition of $50 \mathrm{mM}$ EDTA led to the disappearance of characteristic phosphate bands of $\mathrm{Ca}^{2+}$ phosphate interaction. Note that both citrate and EDTA have absorption bands in this region, the subtractions of which decreased the quality of the spectra.

In DOPS liposomes antisymmetric $\left(\mathrm{v}_{\mathrm{as}} \mathrm{CH}_{2}\right)$ and symmetric $\left(\mathrm{v}_{\mathrm{s}} \mathrm{CH}_{2}\right)$ methylene stretching vibrations dominated at 2924 and $2854 \mathrm{~cm}^{-1}$, respectively (Figure 5. B, spectrum a). 
In control cochleates the methylene stretching band positions shifted towards lower wavenumbers (Figure 5. B, spectrum b) indicating a tighter lipid packing, which may be attributed to the strong interaction of $\mathrm{Ca}^{2+}$ with lipid head groups. Addition of $100 \mathrm{mM}$ citrate had no observable effect in this spectral region (Figure 5. B, spectrum c). However, at a dispersing citrate concentration $(212.5 \mathrm{mM})$ the position of both the asymmetric and symmetric stretching bands shifted to higher wavenumbers approaching those of the $\mathrm{Ca}^{2+}-$ free liposomes (Figure 5. B, spectrum d). Thus, a fraction of lipids became less tightly packed. Upon adding $50 \mathrm{mM}$ EDTA a further increase of $\mathrm{v}_{\mathrm{s}} \mathrm{CH}_{2}$ could be observed as a result of acyl-chain perturbation leading to a lipid bilayer structure, the packing of which is similar to that of liposomes (Figure 5. B, spectrum e).In the spectral region sensitive to ester carbonyl vibrations, DOPS liposomes (Figure 5. C, spectrum a) displayed a broad band that could be deconvoluted into two components (at $\sim 1736 \mathrm{~cm}^{-1}$, and at $\sim 1725 \mathrm{~cm}^{-1}$ ) assigned to differently hydrated subpopulations of the two carbonyl groups [33]. Upon $\mathrm{Ca}^{2+}$ addition, four $\mathrm{vC}=\mathrm{O}$ stretching bands became distinct at 1745, 1729, 1720 and $1704 \mathrm{~cm}^{-1}$ (Figure 5. C, spectrum b). The lower wavenumbers point at the formation of new hydrogen bonds, while band narrowing is due to a significant reduction in motional freedom caused most likely by the immobilization of polar headgroups due to $\mathrm{Ca}^{2+}$ complexation $[34,35]$. Addition of $100 \mathrm{mM}$ citrate did not alter the IR spectrum (Figure 5. B, spectrum c). At the dispersing citrate concentration $(212.5 \mathrm{mM})$, however, the intensities of the narrow $\mathrm{vC}=\mathrm{O}$ stretching bands, peculiar to the $\mathrm{Ca}^{2+}$-DOPS complexes, decreased, and a broad band centered at $1728 \mathrm{~cm}^{-1}$, characteristic to hydrated DOPS lipids, appeared (Figure 6. d). Upon the addition of $50 \mathrm{mM}$ EDTA the carbonyl stretching region of the IR spectrum reverted back to that of DOPS liposomes, and the spectral features characteristic to cochleates disappeared (Figure 6. E). Similar trends were observed in dry film spectra (Supplement figure 8.) with the exception that the narrow $\mathrm{vC}=\mathrm{O}$ stretching bands, specific to $\mathrm{Ca}^{2+}$-bound phospholipids, were more 
dominant even at $212.5 \mathrm{mM}$ citrate (Supplement figure 8. d). Because under dry film conditions the direct interactions are more important, these bands underpin that $\mathrm{Ca}^{2+}$-bound head groups were present even after the addition of $212.5 \mathrm{mM}$ citrate. Altogether the FTIR spectroscopy results indicate that citrate applied in dispersing concentration removes $\mathrm{Ca}^{2+}$ from a fraction of the lipid head groups. Since cochleates with preserved structure prevailed (Figures 2. and 3.) and FTIR spectra also showed the presence of $\mathrm{Ca}^{2+}$-head group complexes in citrate-dispersed samples (Figure 5., spectra d), the $\mathrm{Ca}^{2+}$-deprived fraction may correspond to both the head groups of vesicles and cochleate-surface head groups. Nevertheless, within the dispersing citrate concentration range, the calcium-bound state of the internal lipid head groups of cochleates is left intact. Notably we cannot exclude that some cochleates disintegrated upon addition of citrate at optimal dispersing concentration. This may happen due to local concentration inhomogeneities during mixing the sample with citrate.

\section{Conclusions}

In conclusion, citric acid, an inexpensive, low-molecular-weight and biocompatible material, can be effectively used as a dispersing and stabilizing agent in cochleate nanotechnology. Citrate apparently removes $\mathrm{Ca}^{2+}$ from the cochleate surface rapidly and disrupts calciumdependent cross-links between the particles. Removal of cross-links and an increased electrostatic repulsion between the particles leads to the rapid disassembly of aggregates. By contrast, it takes a longer time for citrate to extract $\mathrm{Ca}^{2+}$ incorporated between the membrane layers. Therefore, the unrolling of cochleates can be prevented by removing excess citrate following aggregate disassembly. Altogether, a disperse cochleate sample with long-term stability is obtained. The citrate-based dispersion and stabilization method is likely to contribute to the development of efficient cochleate-based drug delivery systems. 


\section{Acknowledgements}

This work was supported by grants from the Hungarian Science Foundation (OTKA

K109480) and the National Research, Development and Innovation Office (VKSZ_14-1-

2015-0052). The research leading to these results has received funding from the European

Union's Seventh Framework Program (FP7/2007-2013) under grant agreement ${ }^{\circ}$ HEALTH-

F2-2011-278850 (INMiND) and NMP-2012-309820 (NanoAthero). The FTIR

spectroscopy part was supported by the János Bolyai Research scholarship of the Hungarian Academy of Sciences (J.M.).

Supplementary material is available. 


\section{References}

[1] D. Papahadjopoulos, W.J. Vail, K. Jacobson, G. Poste, Cochleate lipid cylinders: formation by fusion of unilamellar lipid vesicles, Biochim Biophys Acta, 394 (1975) 483 491.

[2] A.D. Bangham, D. Papahadjopoulos, Biophysical properties of phospholipids. I. Interaction of phosphatidylserine monolayers with metal ions, Biochim Biophys Acta Biophysics including Photosynthesis, 126 (1966) 181-184.

[3] A. Martín-Molina, C. Rodríguez-Beas, J. Faraudo, Effect of Calcium and Magnesium on Phosphatidylserine Membranes: Experiments and All-Atomic Simulations, Biophys J, 102 (2012) 2095-2103.

[4] O. Perez, G. Bracho, M. Lastre, N. Mora, J. del Campo, D. Gil, C. Zayas, R. Acevedo, D. Gonzalez, J.A. Lopez, C. Taboada, C. Turtle, R.L. Solis, Novel adjuvant based on a proteoliposome-derived cochleate structure containing native lipopolysaccharide as a pathogen-associated molecular pattern, Immunol Cell Biol, 82 (2004) 603-610.

[5] J. Del Campo, M. Lindqvist, M. Cuello, M. Backstrom, O. Cabrerra, J. Persson, O. Perez, A.M. Harandi, Intranasal immunization with a proteoliposome-derived cochleate containing recombinant $\mathrm{gD}$ protein confers protective immunity against genital herpes in mice, Vaccine, 28 (2010) 1193-1200.

[6] B. Romeu, E. Gonzalez, J. Del Campo, R. Acevedo, C. Zayas, Y. Valdes, O. Cabrera, M. Cuello, J. Balboa, M. Lastre, O. Perez, Mucosal and systemic immune responses of mice to tetanus toxoid coadministered nasally with AFCo1, Can J Microbiol, 57 (2011) 256-261. [7] C. Zayas, D. González, R. Acevedo, J. del Campo, M. Lastre, E. González, B. Romeu, M. Cuello, J. Balboa, O. Cabrera, L. Guilherme, O. Pérez, Pilot scale production of the vaccine adjuvant Proteoliposome derived Cochleates (AFCo1) from Neisseria meningitidis serogroup B, BMC Immunology, 14 (2013) 1-5.

[8] N. Wang, T. Wang, M. Zhang, R. Chen, Y. Deng, Using procedure of emulsificationlyophilization to form lipid A-incorporating cochleates as an effective oral mucosal vaccine adjuvant-delivery system (VADS), International Journal of Pharmaceutics, 468 (2014) 39-49. [9] L. Livne, R.F. Epand, B. Papahadjopoulos-Sternberg, R.M. Epand, A. Mor, OAK-based cochleates as a novel approach to overcome multidrug resistance in bacteria, FASEB J, 24 (2010) 5092-5101.

[10] H. Sarig, D. Ohana, R.F. Epand, A. Mor, R.M. Epand, Functional studies of cochleate assemblies of an oligo-acyl-lysyl with lipid mixtures for combating bacterial multidrug resistance, FASEB J, 25 (2011) 3336-3343.

[11] R.F. Epand, H. Sarig, D. Ohana, B. Papahadjopoulos-Sternberg, A. Mor, R.M. Epand, Physical properties affecting cochleate formation and morphology using antimicrobial oligoacyl-lysyl peptide mimetics and mixtures mimicking the composition of bacterial membranes in the absence of divalent cations, J Phys Chem B, 115 (2011) 2287-2293.

[12] R.F. Epand, A. Mor, R.M. Epand, Lipid complexes with cationic peptides and OAKs; their role in antimicrobial action and in the delivery of antimicrobial agents, Cell Mol Life Sci, 68 (2011) 2177-2188.

[13] R. Santangelo, P. Paderu, G. Delmas, Z.W. Chen, R. Mannino, L. Zarif, D.S. Perlin, Efficacy of oral cochleate-amphotericin B in a mouse model of systemic candidiasis, Antimicrob Agents Chemother, 44 (2000) 2356-2360.

[14] L. Zarif, J.R. Graybill, D. Perlin, L. Najvar, R. Bocanegra, R.J. Mannino, Antifungal activity of amphotericin B cochleates against Candida albicans infection in a mouse model, Antimicrob Agents Chemother, 44 (2000) 1463-1469. 
[15] I. Segarra, D.A. Movshin, L. Zarif, Pharmacokinetics and tissue distribution after intravenous administration of a single dose of amphotericin B cochleates, a new lipid-based delivery system, J Pharm Sci, 91 (2002) 1827-1837.

[16] G. Delmas, S. Park, Z.W. Chen, F. Tan, R. Kashiwazaki, L. Zarif, D.S. Perlin, Efficacy of orally delivered cochleates containing amphotericin B in a murine model of aspergillosis, Antimicrob Agents Chemother, 46 (2002) 2704-2707.

[17] R.D. Miclea, P.R. Varma, A. Peng, S.V. Balu-Iyer, Development and characterization of lipidic cochleate containing recombinant factor VIII, Biochim Biophys Acta, 1768 (2007) 2890-2898.

[18] M.P. Kosloski, A. Peng, P.R. Varma, A.M. Fathallah, R.D. Miclea, D.E. Mager, S.V. Balu-iyer, Immunogenicity and pharmacokinetic studies of recombinant factor VIII containing lipid cochleates, Drug Deliv, 18 (2011) 246-254.

[19] R.J. Mannino, S. Gould-Fogerite, S.L. Krause-Elsmore, D. Delmarre, R. Lu, Novel encochleation methods, cochleates and methods of use, in, Google Patents, 2004.

[20] K. Nagarsekar, M. Ashtikar, F. Steiniger, J. Thamm, F.H. Schacher, A. Fahr, Microspherical cochleate composites: method development for monodispersed cochleate system, J Liposome Res, (2016) 1-9.

[21] S. Nir, J. Bentz, J. Wilschut, N. Duzgunes, Aggregation and fusion of phospholipid vesicles, Prog Surf Sci, 13 (1983) 1-124.

[22] A. Wacha, Z. Varga, A. Bota, CREDO: a new general-purpose laboratory instrument for small-angle X-ray scattering, J Appl Crystallogr, 47 (2014) 1749-1754.

[23] A. Wacha, Optimized pinhole geometry for small-angle scattering, J Appl Crystallogr, 48 (2015) 1843-1848.

[24] N.R. Joseph, The dissociation constants of organic calcium complexes, J Biol Chem, 164 (1946) 529-541.

[25] R.N. Goldberg, N. Kishore, R.M. Lennen, Thermodynamic Quantities for the Ionization Reactions of Buffers, J Phys Chem Ref Data, 31 (2002) 231-370.

[26] R.K. Cannan, A. Kibrick, Complex Formation between Carboxylic Acids and Divalent Metal Cations, J Am Chem Soc, 60 (1938) 2314-2320.

[27] G. Arena, S. Musumeci, R. Purrello, S. Sammartano, Calcium- and magnesium-EDTA complexes. Stability constants and their dependence on temperature and ionic strength, Thermochim Acta, 61 (1983) 129-138.

[28] A.E.M. Robert M. Smith, Critical Stability Constants, Plenum Press, New York, 1989.

[29] T. Bozo, R. Brecska, P. Grof, M.S. Kellermayer, Extreme resilience in cochleate nanoparticles, Langmuir, 31 (2015) 839-845.

[30] A. Hollander, D. Danino, Cochleate characterization by cryogenic electron microscopy methods: Cryo-TEM and Cryo-SEM, Colloids Surface A, 483 (2015) 187-192.

[31] M.R. Brzustowicz, A.T. Brunger, X-ray scattering from unilamellar lipid vesicles, Journal of Applied Crystallography, 38 (2005) 126-131.

[32] R. Dluhy, D.G. Cameron, H.H. Mantsch, R. Mendelsohn, Fourier transform infrared spectroscopic studies of the effect of calcium ions on phosphatidylserine, Biochemistry, 22 (1983) 6318-6325.

[33] R.N. Lewis, R.N. McElhaney, W. Pohle, H.H. Mantsch, Components of the carbonyl stretching band in the infrared spectra of hydrated 1,2-diacylglycerolipid bilayers: a reevaluation, Biophys J, 67 (1994) 2367-2375.

[34] H.L. Casal, H.H. Mantsch, H. Hauser, Infrared studies of fully hydrated saturated phosphatidylserine bilayers. Effect of lithium and calcium, Biochemistry, 26 (1987) 44084416.

[35] H.L. Casal, H.H. Mantsch, F. Paltauf, H. Hauser, Infrared and 31P-NMR studies of the effect of $\mathrm{Li}+$ and $\mathrm{Ca} 2+$ on phosphatidylserines, Biochim Biophys Acta, 919 (1987) 275-286. 


\section{Figures}
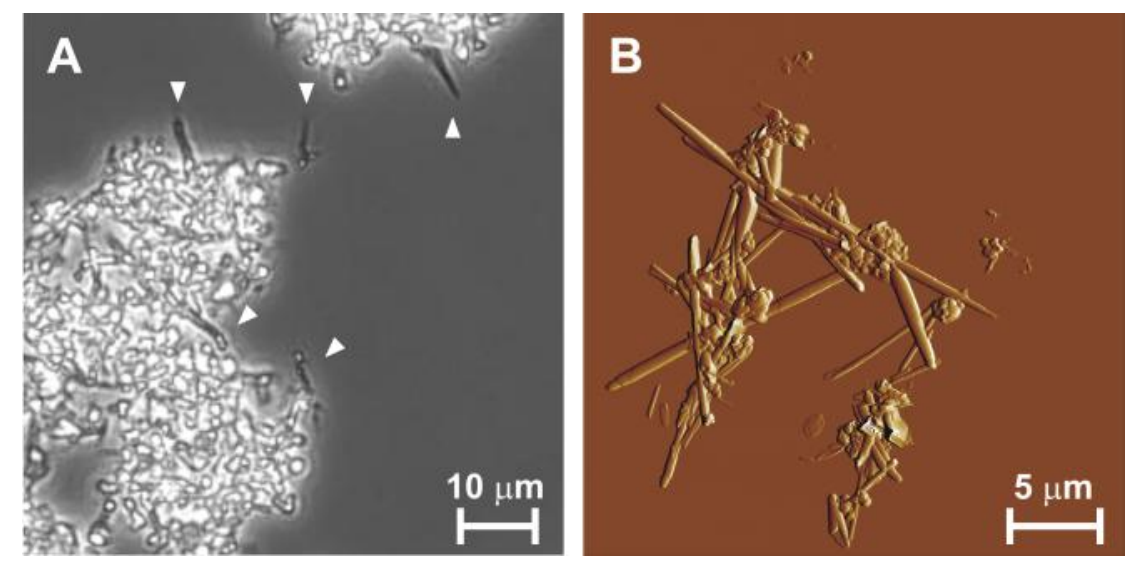

Figure 1. Structure of nascent cochleates. (A) Phase contrast micrograph of dialyzed cochleates. White arrowheads point at elongated structures identified as cochleates. (B) Amplitude-contrast AFM image of a cochleate aggregate.
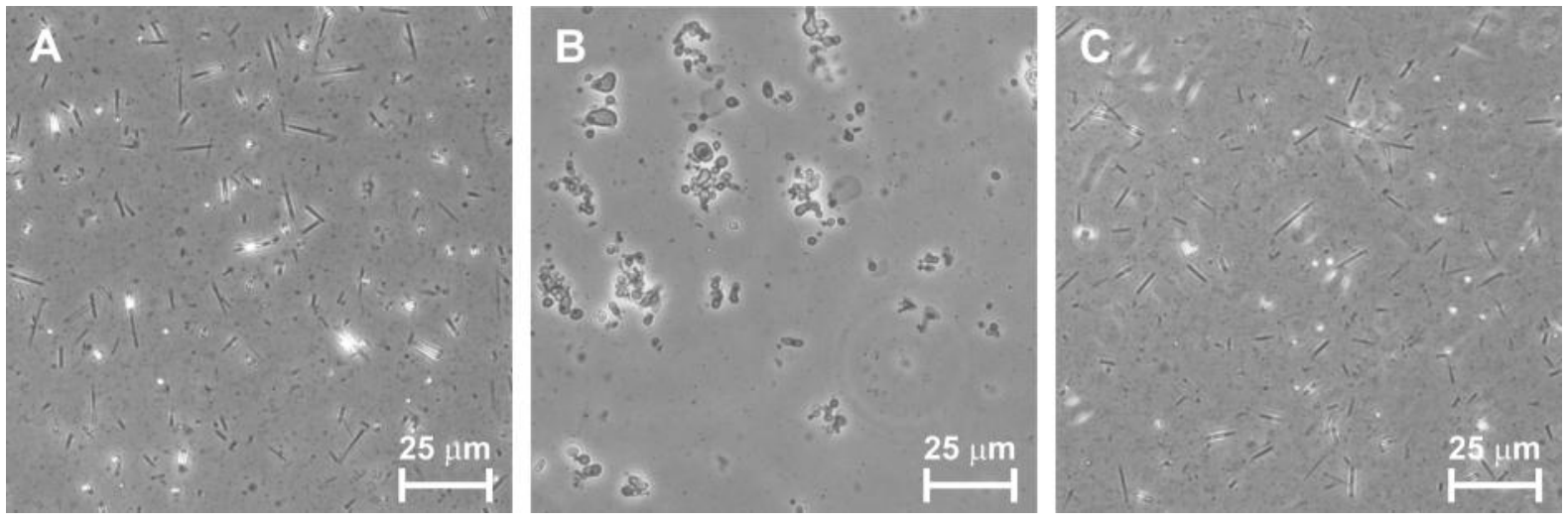

Figure 2. Phase contrast micrographs of citrate-treated cochleate samples. (A) Cochleates dispersed in $212.5 \mathrm{mM}$ sodium citrate. (B) The same sample after $24 \mathrm{~h}$ of incubation at room temperature. (C) Same sample as in (A) but centrifuged and resuspended in $100 \mathrm{mM}$ TRIS $\mathrm{pH}=7.4$ buffer immediately following citrate treatment. 

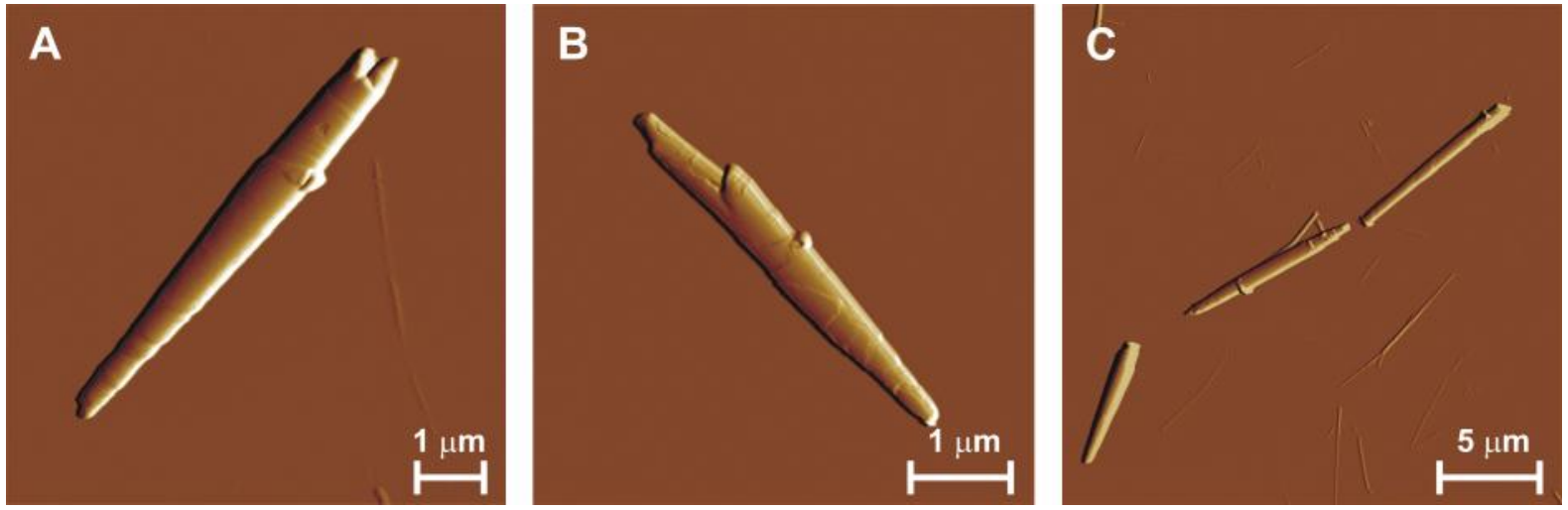

Figure 3. AFM amplitude images of a citrate-stabilized cochleates.

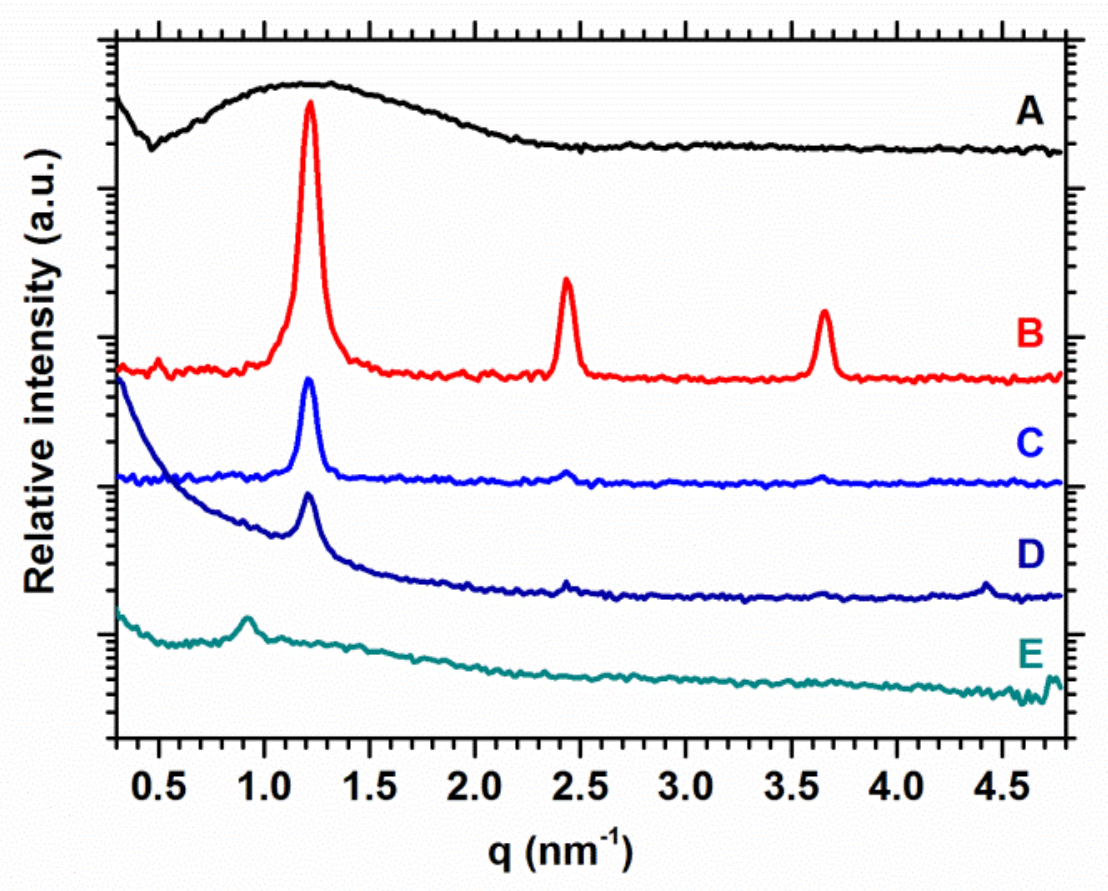

Figure 4. SAXS diffractograms of (A) DOPS MLV-s, (B) control cochleates, (C) cochleates $+100 \mathrm{mM}$ citrate, $(\mathrm{D})$ cochleates $+212.5 \mathrm{mM}$ citrate, $(\mathrm{E})$ cochleates $+50 \mathrm{mM}$ EDTA.

Diffractograms are gradually shifted along relative intensity axis for better display. 
A

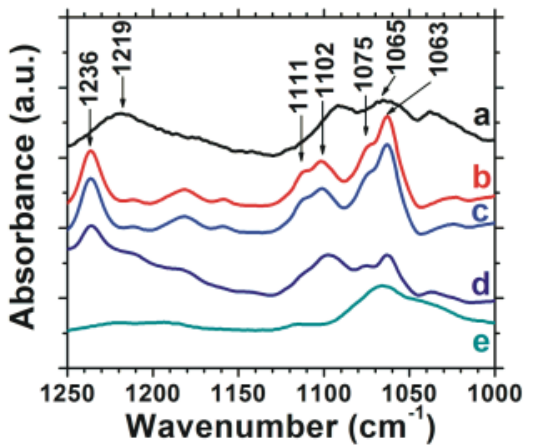

B

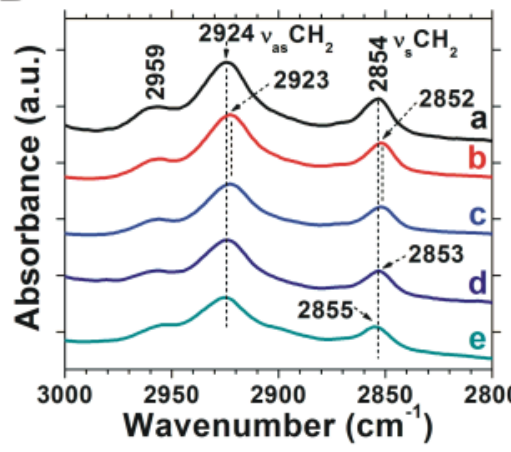

C

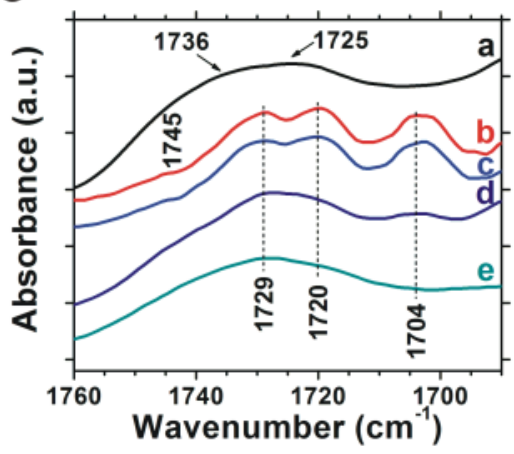

Figure 5. The (A) phosphate, (B) C-H and (C) ester carbonyl stretching region of the infrared spectra of hydrated lipid systems (corresponding buffer spectra are subtracted). (a) DOPS SUV, (b) cochleates; (c) cochleates $+100 \mathrm{mM}$ citrate, $(\mathrm{d})$ cochleates $+212.5 \mathrm{mM}$ citrate; (e) cochleates + 50 mM EDTA. 